\title{
Saint-André de Bâgé
}

Etang Quinard et Pré des Mouilles - Autoroute 406 -contournement sud de Mâcon

\section{Cécile Ramponi}

\section{(Q) OpenEdition Journals}

Édition électronique

URL : http://journals.openedition.org/adlfi/3475

ISSN : 2114-0502

Éditeur

Ministère de la culture

Référence électronique

Cécile Ramponi, «Saint-André de Bâgé », ADLFI. Archéologie de la France - Informations [En ligne], Rhône-Alpes, mis en ligne le 01 mars 2009, consulté le 19 avril 2019. URL : http://

journals.openedition.org/adlfi/3475

Ce document a été généré automatiquement le 19 avril 2019

(C) Ministère de la Culture et de la Communication, CNRS 


\section{Saint-André de Bâgé}

Etang Quinard et Pré des Mouilles - Autoroute 406 -contournement sud de Mâcon

Cécile Ramponi

Identifiant de l'opération archéologique : 10051

Date de l'opération : 2009 (SP)

1 L'opportunité d'un diagnostic, de $7 \mathrm{~km}$ de long, sur la rive gauche de la Saône, au droit de la ville de Mâcon, avait permis de repérer des implantations humaines, de la fin de l'âge du Bronze (urnes cinéraires à Crottet), du début de l'âge du Fer (fosse à Replonges les Platières), de la fin de la période de La Tène (Saint-André-de Bâgé et Replonges) et de la période gallo-romaine (Replonges et Crottet) (Ramponi et Franc, 2008). Cette petite portion de territoire, mal connue archéologiquement, semblait relativement peu fréquentée au cours de la Pré-Protohistoire ainsi qu'à l'époque gallo-romaine. Ce vide semble en partie du à l'absence d'interventions archéologiques. En effet les dernières interventions sur ce secteur tendent à montrer que l'homme, au cours des siècles, s'est installé sur le plateau bressan mais également dans la plaine alluviale de la Saône (occupation de l'âge du Bronze à Grièges : Vermeulen, Attiah, Vérot-Bourrely, 2007 ).

2 Les résultats de la fouille effectuée aux lieux-dits Etang Quinard et Pré des Mouilles ne sont pas aussi fournis que ce que laissait espérer la campagne de diagnostic. Pour autant, ils ne sont pas nuls et permettent pour la première fois avec la fouille de Replonges Les Platières - de mettre en évidence non seulement une fréquentation du lieu et des installations humaines de l'âge du Bronze (ou du Néolithique ?), du milieu de l'âge du Fer (VI ${ }^{\mathrm{e}}$. - $\mathrm{V}^{\mathrm{e}} \mathrm{s}$. av. J.-C) et de la fin de la période de La Tène.

\section{Le Néolithique et l'âge du Bronze}

3 Un petit aménagement de galets thermofractés, en partie démantelé, a été retrouvé dans une couche antérieure à l'occupation de l'âge du Fer. Sa véritable fonction reste assez 
vague (structure en place ou vidange de foyer) mais la concentration des éléments plaide en faveur de la première proposition. La présence de chutes de taille de silex, de mobilier de mouture et de céramiques pourrait indiquer qu'elle est liée à des activités de la vie quotidienne. Étant malheureusement isolée en bordure de site nous ne pouvons expliciter son intégration dans une occupation plus large. S'agit-il d'une installation pérenne ou ponctuelle, isolée ou intégrée à un habitat? Enfin, quelle forme avait cet habitat? De plus, le mobilier céramique, qui ne présente aucune forme ni aucun décor, reste trop indéfini pour permettre une datation plus resserrée que celle couvrant la fin de la période néolithique et le début de l'âge du Bronze.

\section{Le premier âge du Fer}

4 Le niveau d'amphore de Marseille retrouvé lors du diagnostic reste isolé et aucun aménagement ne peut lui être associé. Toutefois, cette découverte est suffisamment rare pour être soulignée. A ce jour, dans les publications, le département de l'Ain ne compte qu'un seul site en ayant livré - la grotte du Gardon (Buisson 1990, p. 24). Il s'agit d'un site en grotte et les sites de plein-air les plus proches connus à ce jour sont celui de Bragny en Saône-et-Loire et ceux de Lyon-Vaise et Saint-Priest Les Luêpes et Les Feuilly dans le Rhône. Ces sites sont de natures et d'ampleurs différentes. La présence de matériel d'importation - amphore et céramique, plus rarement de céramique attique - les place sur les voies de commerce reliant les comptoirs méditerranéens aux sites septentrionaux. Saint-André-de-Bâgé se trouve sur un de ces axes commerciaux supposés, empruntant la vallée du Rhône puis de la Saône.

\section{La fin de l'âge du Fer}

5 C'est pour cette période que les découvertes sont les plus significatives. Elles restent toutefois limitées à des plans de bâtiments, une fosse et une sole de foyer. Les bâtiments sur poteaux au nombre de 4 ( 2 certains et 2 incomplets) sont des constructions de type grenier. L'un d'entre eux est construit à l'aide de doubles poteaux. Les espaces supportés, hors plateforme éventuelle, s'étalent de 8 à $14 \mathrm{~m}^{2}$. La comparaison avec les sites de Sennecé-lès-Mâcon «En Putet » et Replonges «les Platières » indique des superficies similaires mais avec des avant-trous et des poteaux de diamètres supérieurs.

6 La majorité du mobilier, retrouvé dans une fosse et dans le décapage (78 \% du corpus) date l'installation de La Tène finale. La répartition de ce mobilier se concentre dans les secteurs nord et sud-ouest de l'emprise de fouille, laissant penser que le site se poursuit dans ces directions. La partie centrale, occupée par les bâtiments, est exempte de mobilier, suggérant qu'il ne s'agit pas d'une zone d'activité quotidienne.

7 La nature des installations ainsi que la faible quantité de mobilier et de rejets retrouvés ne nous apportent pas d'indices très probants sur la fonction du site. Seuls les bâtiments sur poteaux attestent une occupation pérenne. Nous sommes probablement en périphérie de site, dans une zone dédiée au stockage à priori éloignée des zones d'activités artisanales ou de la vie quotidienne. Nous ne pouvons juger de l'importance ni de la durée d'occupation $\mathrm{du}$ site. Les quelques éléments céramiques attribués à la période augustéenne pourraient indiquer, mais sans certitudes, un terminus post-quem à l'installation. 


\section{Les périodes historiques}

8 Malgré la présence d'un peu de mobilier augustéen, le site n'a révélé aucune occupation gallo-romaine. Les périodes postérieures ne sont pas du tout représentées et seul un réseau de fossés, dont l'ancienneté ne peut être démontrée, témoignent de l'emprise de l'homme sur le paysage aux périodes historiques.

9 RAMPONI Cécile

INDEX

operation Sauvetage programmé (SP)

Index chronologique : âge du Bronze, âge du Fer, La Tène III, Néolithique

Thèmes : amphore, céramique attique, débitage, foyer, galet aménagé, habitat, silex, trou de poteau

Index géographique : Rhône-Alpes, Ain (01), Saint-André-de-Bâgé

\section{AUTEUR}

CÉCILE RAMPONI

INRAP 